\title{
Plasma Free and Sulfoconjugated Dopamine before and after a Half-Marathon
}

\author{
Takaaki Sakai, Hirokazu Maeda, Naomichi Matsumoto, Shin-ichiro Miura, \\ Akio Kinoshita, Manabu Sasaguri, Munehito Ideishi, Hiroaki Tanaka*, \\ Munehiro Shindo*, and Kikuo Arakawa
}

\begin{abstract}
To elucidate whether increased plasma levels of free dopamine (F-DA) after exercise are due to deconjugation of sulfoconjugated (S-) DA in plasma, we compared the changes in plasma F- and S- DA, as well as changes in both the $S$ - and $F$ - forms of epinephrine (E) and norepinephrine (NE), after running a half-marathon. Free catecholamines (F-CAs) were measured by automated high-performance liquid chromatography (HPLC). Total $(F+S) C A s$ were determined using an efficient deconjugation method as follows; $1200 \mu$ l plasma was incubated with $152 \mathrm{mU}$ arylsulfatase (AS) for $30 \mathrm{~min}$ at pH 7.6. The plasma levels of F-CA $(\mathrm{pg} / \mathrm{ml})($ mean \pm SEM) all increased significantly $(p<0.01)$ after the half-marathon: i.e., F-DA increased from $13.3 \pm 5.7$ to $176.3 \pm 32.2$; F-E from $58.0 \pm 12.3$ to $764.3 \pm 136.4$; F-NE from 246.6 \pm 15.2 to $3082.0 \pm 690.3$. Of S-CAs, S-E (from 127.8 \pm 26.0 to $1218.2 \pm 190.8$ ) and S-NE (from $717.1 \pm 61.6$ to $5586.9 \pm 761.9)$ also increased, but, in contrast, among the S-CAs, only the increase in SDA (from 5324.9 \pm 1967.3 to $7359.6 \pm 1627.9$ ) was not statistically significant. Sulfoconjugation may play an important role in inactivating F-DA as well as F-NE and -E, that are released into plasma in response to vigorous exercise. Thus, plasma F-DA is unlikely to be derived through deconjugation of plasma S-DA. (Hypertens Res 1995; 18 Suppl. I: S161-S163)
\end{abstract}

Key Words: free dopamine, sulfoconjugated dopamine, arylsulfatase, physical exercise

Catecholamines (CA) in human plasma contribute to the regulation of circulatory homeostasis. It has been reported that physical exercise influences the level of plasma free (F-) and sulfoconjugated (S-) CAs (1). F-CAs are known to be inactivated by three major metabolic pathways, i.e. deamination, O-methylation and conjugation (2). Most CAs in human plasma exist in the sulfoconjugated form, while only a small portion is in the glucuronide form. Sixty to $70 \%$ of plasma epinephrine (E) and norepinephrine (NE) is conjugated, whereas more than $98 \%$ of the dopamine (DA) in human plasma is in the conjugated form $(3,4)$. S-CAs, especially $\mathrm{S}-\mathrm{DA}$, have been reported to be converted to $\mathrm{F}$ CAs in response to physical exercise $(5,6)$. A recently developed automated HPLC analyzer has made it possible to quantify F-DA with great accuracy (7).

To examine whether increased plasma levels of FDA after exercise are due to deconjugation of SDA in plasma, plasma F- and S-DA, as well as both the S- and F-forms of $\mathrm{E}$ and $\mathrm{NE}$, were measured before and after a half-marathon using an automated high-performance liquid chromatography (HPLC) analyzer with or without treatment by arylsulfatase (AS).

\section{Materials and Methods}

Determination of the Optimal Conditions for Deconjugation of $S-C A$

Plasma samples $(1,200 \mu \mathrm{l})$ were incubated in $0.01 \mathrm{M}$ Tris buffer $(\mathrm{pH} \mathrm{7.6)}$ with various concentrations of AS $(0,19,38,76,152,304,608 \mathrm{mU})$ (Sigma, Type VI) in a total volume of $1,300 \mu \mathrm{l}$. After incubation at $37^{\circ} \mathrm{C}$ for a given duration $(0,5,10,15,25,40$ $\min ), 600 \mu \mathrm{l}$ of a solution consisting of $0.35 \mathrm{M}$ sodium acetate and $6 \%$ perchloric acid was added to stop the reaction. The efficiency of deconjugation by $\mathrm{AS}$ at $\mathrm{pH} 7.6$ (optimal $\mathrm{pH}$ for AS) was compared to that at $\mathrm{pH} 8.3$ (optimal $\mathrm{pH}$ for COMT) using paired plasma samples from normal volunteers. After centrifugation $(11,750 \times g)$ at $4^{\circ} \mathrm{C}$ for $20 \mathrm{~min}$, the supernatant was applied to an HPLC analyzer (TSK gel CatecholPak, Tosoh Co, Tokyo, Japan) (6). S-CA was obtained by subtracting F- from total (T)-CA (measured after deconjugation).

\section{Subjects}

The study group consisted of 9 healthy male volunteers, aged 21 to 46 years old, who ran a city halfmarathon (Fukuoka City Marathon, Fukuoka). They were not taking any drugs and showed no

From the Department of Internal Medicine, School of Medicine, and * Department of Exercise Physiology, School of Physical Education, Fukuoka University, Fukuoka, Japan.

Address for Reprint: Takaaki Sakai, M.D., Department of Internal Medicine, School of Medicine, Fukuoka University, 7-45-1 Nanakuma, Jonan-ku, Fukuoka 814-80, Japan. 

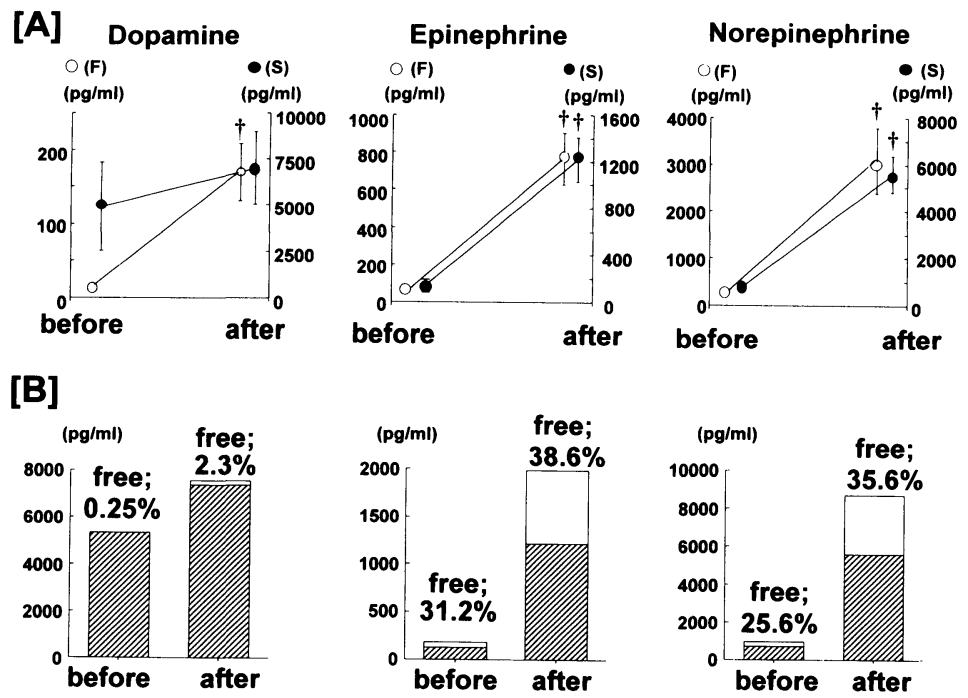

Fig. 1. Plasma concentration of free $(F)$ and sulfoconjugated $(S)$ catecholamines $[A]$, and the percentage ratio of free to total catecholamines [B] before and after a half-marathon. Values are mean $\pm S E M .{ }^{\dagger}$ indicates $\mathrm{p}<0.01:$ Significant difference between before and after. $\square$, free; $\mathbb{Z}$, sulfoconjugated catecholamines.

abnormalities in routine laboratory examinations. Although they were allowed to walk or stop for a few minutes whenever they felt tired, they all completed the half-marathon. Most of the subjects were habitual, but not competitive, runners. They were thoroughly informed of the nature of this study, and their informed consent was obtained prior to their participation.

\section{Preparation of Plasma Samples}

With the subject in a sitting position, $14 \mathrm{ml}$ of blood was drawn from a cubital vein before and immediately after the half-marathon. The blood samples were transferred to an ice-chilled centrifuge tube containing $10 \mathrm{mg}$ EDTA-2Na. After the blood was mixed by gentle inversion, the plasma was separated from the blood cells by centrifugation $(11,750 \times g)$ at $4^{\circ} \mathrm{C}$ for $10 \mathrm{~min}$, and then stored at $-70^{\circ} \mathrm{C}$ until assay.

\section{Statistics}

Values are expressed as the mean \pm SEM for both experimental and clinical studies. Student's $t$ test was used for statistical analysis, and $p<0.01 v s$. the control basal value was considered significant.

\section{Results}

\section{Deconjugation of $S$ - $C A$}

S-CAs reached a plateau after incubation for up to $30 \mathrm{~min}$, and $152 \mathrm{mU}$ AS was sufficient for deconjugation. More S-DA was deconjugated at $\mathrm{pH} 7.6$ (optimal $\mathrm{pH}$ for $\mathrm{AS}$ ) than at $\mathrm{pH} 8.3$ (optimal $\mathrm{pH}$ for COMT) $(2684.4 \pm 298.0 \mathrm{pg} / \mathrm{ml}$ at $\mathrm{pH} 7.6 \mathrm{vs}$. $1462.4 \pm 190.8 \mathrm{pg} / \mathrm{ml}$ at $\mathrm{pH} 8.3)$. Total $\mathrm{E}$ and $\mathrm{NE}$ also tended to be higher at $\mathrm{pH} 7.6$ than that at $\mathrm{pH}$ 8.3 (E: $116.7 \pm 12.7 \mathrm{pg} / \mathrm{ml}$ at $\mathrm{pH} 7.6$ vs. $89.0 \pm 16.7$ $\mathrm{pg} / \mathrm{ml}$ at $\mathrm{pH} 8.3$, NE: $995.4 \pm 185.9 \mathrm{pg} / \mathrm{ml}$ at $\mathrm{pH} 7.6$ vs. $667.8 \pm 150.2 \mathrm{pg} / \mathrm{ml}$ at $\mathrm{pH} 8.3$ ).
Change in Plasma CA after the Half-Marathon

The plasma levels of F- DA, -E and -NE ( $\mathrm{pg} / \mathrm{ml})$ increased significantly $(p<0.01)$ after the halfmarathon (DA; from $13.3 \pm 5.7$ to $176.3 \pm 32.2$, E; from $58.0 \pm 12.3$ to $764.3 \pm 136.4, \mathrm{NE}$; from $246.6 \pm$ 15.2 to $3082.0 \pm 690.3)$. S-E and $-\mathrm{NE}$ also increased significantly (E; from $127.8 \pm 26.0$ to $1218.2 \pm 190.8$, $\mathrm{NE}$; from $717.1 \pm 61.6$ to $5586.9 \pm 761.9)(p<0.01)$. In contrast, although S-DA increased, this increase was not significant (from 5324.9 \pm 1967.3 to 7359.6 \pm 1627.9 ) (Fig. $1 \mathrm{~A}$ ). The percentage ratio of free $\mathrm{CA}$ to total CA increased after the half-marathon and the percentage increase was greater in DA (from $0.25 \%$ to $2.3 \%$ ) than in $\mathrm{E}$ or $\mathrm{NE}$ (E: from $31.2 \%$ to $38.6 \%$, NE: from $25.6 \%$ to $35.6 \%$ ) (Fig. 1B).

\section{Discussion}

The aim of this study was to elucidate whether increased plasma F-DA is derived from S-DA in plasma. We examined the changes in plasma levels of F- and S-DA after a half-marathon. To measure these levels more accurately than has been previously reported, we quantified total DA using an HPLC analyzer after deconjugation at $\mathrm{pH} 7.6$ by enzymatic hydrolysis with AS. The conjugated DA at rest in our study was greater than those in previous reports probably because we deconjugated at the optimal $\mathrm{pH}$ of 7.6 for AS, instead of at $\mathrm{pH} 8.3$. In most previous studies, CAs were measured as metabolites by radioenzymatic assay after treatment with COMT, and deconjugation was performed at $\mathrm{pH} 8.3$, which is optimal for COMT, but not for AS $(5,7,8)$. This may explain why the ratio of F-DA to T-DA in our study $(0.25 \%)$ was lower than those $(0.5 \%$ or $1 \%)$ in previous reports.

In this study, we have shown that the plasma levels of F-DA, -E and -NE increased significantly 
after the half-marathon. S-E and -NE also increased significantly. On the other hand, although S-DA increased, this increase was not significant. Contrary to our findings, Yoshizumi et al. have reported that F-CAs increased significantly, while S-DA and SNE decreased significantly, after a marathon and suggested the possibility that the increase in plasma F-CAs was due not only to increased release from the sympathoadrenal system, but also to acceleration of deconjugation of S-CAs, especially S-DA (5). Independently, Ratge et al. also reported that F-E and -NE increased significantly along with significant decreases in both S-E and -NE in plasma at maximum workload during brief vigorous bicycle exercise, while neither the S- nor F- forms of DA changed. They concluded that S-E and -NE may be used as pools for F-E and -NE during vigorous exercise (6). In these two reports, plasma CAs were measured by radioenzymatic assay, and deconjugation by $\mathrm{AS}$ was not performed at its optimal $\mathrm{pH}$. These differences in technique may explain the conflicting results.

In our study, F-CAs, i.e. F-DA, -E, and -NE, in plasma increased to the same extent, approximately 13-fold. On the other hand, the plasma levels of S$E$ increased about 9.5-fold, and that of S-NE increased about 7.8-fold. In contrast, S-DA increased only about 1.4-fold. The different increase in S-CAs might be due to the fact that less plasma DA was released from the sympathoadrenal system in response to vigorous exercise than $\mathrm{E}$ and $\mathrm{NE}$, suggesting that F-DA may be released differently from dopaminergic neurons. Our study demonstrated that conjugation of F-CAs may play an important role in the inactivation of newly released F-CAs. Concerning DA, we could not exclude the possibility that a small portion of F-DA was converted from S-DA because the ratio of F-DA to T-DA increased approximately 10 -fold while those of $\mathrm{E}$ and $\mathrm{NE}$ increased only slightly. However, F-DA is readily conjugated compared to F-E and -NE, since phenolsulfotransferase (PST) is reported to have a much higher affinity for F-DA than for F-NE or -E, which contributes to the greater percentage of $S$ DA (9). The higher ratio of F-DA to T-DA might suggest that F-DA is released and conjugated later in the half-marathon than F-E and -NE. In this study, we measured plasma levels of F- and S-CAs at only two points; before and after exercise. To further clarify the kinetics of F- and S-CAs, quan- tification of plasma levels of F- and S-CAs at different points during exercise and at different exercise intensities will be needed.

In summary, our study has shown that sulfoconjugation may play an important role in the inactivation of F-DA as well as F-NE and -E released in response to vigorous exercise. Thus, plasma F-DA is unlikely to be derived through deconjugation of plasma S-DA. We also have demonstrated that the deconjugation of S-CAs by AS treatment at $\mathrm{pH} 7.6$ combined with HPLC is superb method to determine plasma levels of DA.

\section{Acknowledgements}

We thank Miss Y.Saito for her skillful technical assistance and R. Hayashi for providing secretarial services.

\section{References}

1. Joyce DC, Beilin LJ, Vandongen R, Davidson L: Plasma free and sulfate-conjugated catecholamine levels during acute physiological stimulation in man. Life Sci 1982; 30: 447-454.

2. Kopin IJ: Catecholamine metabolism: Basic aspects and clinical significance. Pharmacol. Rev 1983; 37: 333-364

3. Johnson GA, Baker CA, Smith RT: Radioenzymatic assay of sulfate conjugates of catecholamines and dopa in plasma. Life Sci 1980; 26: 1591-1598.

4. Oka M, Ishimura Y, Tsumematsu T, Minakuchi K, Ohuchi T, Matsumoto K: Effects of administration of dopamine and L-dopa to dogs on their plasma level of dopamine sulfate. Biochem. Pharmacol. 1987; 36: 3205-3208.

5. Yoshizumi M, Nakaya Y, Oka M, et al: Changes in plasma free and sulfoconjugated catecholamines before and after acute physical exercise: Experimental and Clinical Studies. Life Sci 1992; 51: 227-234.

6. Ratge D, Gehrke A, Melzner I, Wisser H: Free and conjugated catecholamines in human plasma during physical exercise. Clin Exp Pharmacol Physiol 1986; 13: 543-553.

7. Iwaeda T, Kuroki M, Ohta K, Ishimura S,Takahashi $\mathrm{H}$, Watanabe $\mathrm{H}$ : Development of fully automated catecholamine analyzer, HLC-8030. $J$ Tosoh Research 1988; 32: 59-64.

8. Cléroux J, Péronnet F, Champlain J: Free and conjugated catecholamines in plasma and erythrocytes during exercise and following recovery. Med Sci Sports Exerc 1983; 15: 95. (Abstract)

9. Van Loon GR: Plasma dopamine: regulation and significance. Fed Proc 1983; 42: 3012-3018. 